\title{
The relationship between severity of disease and vitamin D levels in children with atopic dermatitis
}

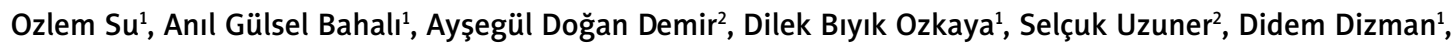 \\ Nahide Onsun ${ }^{1}$
}

'Department of Dermatology, Medical Faculty, Bezmialem Vakif University, Istanbul, Turkey

${ }^{2}$ Department of Pediatrics, Medical Faculty, Bezmialem Vakif University, Istanbul, Turkey

Adv Dermatol Allergol 2017; XXXIV (3): 224-227

DOI: https://doi.org/10.5114/pdia.2017.66054

\begin{abstract}
Introduction: Atopic dermatitis (AD) is a chronic relapsing inflammatory skin disorder. Vitamin D is a liposoluble vitamin synthesized mainly in the skin. Vitamin $D$ has several effects on the skin.

Aim: To assess the serum level of vitamin $D$ in children with $A D$ and determine its relation to $A D$ severity.

Material and methods: Sixty patients with AD were enrolled in the study. We evaluated disease severity using the SCORing Atopic Dermatitis (SCORAD) index. The control group consisted of 37 healthy subjects.

Results: The mean serum concentration of $25(\mathrm{OH}) \mathrm{D}_{3}$ in patients with $A D$ was not statistically different from control subjects $(p=0.065)$. The vitamin $\mathrm{D}$ level was significantly lower in moderate and severe AD compared with mild $A D$, and this difference was statistically significant $(p=0.001$ and $p=0.004)$. Vitamin $D$ showed a negative correlation with serum total IgE levels $(p=0.007)$. There was no significant correlation between total IgE levels and SCORAD scores $(p=0.089)$.

Conclusions: This study suggests that a low serum vitamin $D$ level is inversely associated with severity of $A D$ in children. Vitamin D has not been included in the routine treatment of AD because of the conflicting results of various studies. Thus, there is a need for more detailed and prospective studies.
\end{abstract}

Key words: atopic dermatitis, vitamin D, SCORing Atopic Dermatitis.

\section{Introduction}

Atopic dermatitis (AD) is a chronic relapsing inflammatory skin disorder. It is characterized by pruritus, eczematous plaques, and a defective skin barrier. The ethiopathogenesis of AD is multicomplex and not yet fully understood. New concepts have emerged that demonstrate immunological and inflammatory dysfunction in $A D$, in addition to the importance of environmental factors.

Vitamin D is a liposoluble vitamin synthesized mainly in the skin. It is involved in important regulatory mechanisms of the innate and adaptive immune system, in addition to its roles in calcium homeostasis and bone metabolism. It also has several effects on the skin, including normal keratinocyte development and function, increased wound-healing, decreased inflammation, and the stimulation of protein synthesis, such as filaggrin, which is necessary for stratum corneum barrier formation [1-3].
Recently, there have been several reports that low vitamin D plays a role in the pathogenesis of many conditions, such as autoimmune diseases, infections, malignancies, cardiovascular disease, neuropsychiatric disorders, and allergic diseases, including AD [4-6]. Several studies have reported on vitamin $D$ levels in $A D$ patients, vitamin $D$ supplementation in the treatment of $A D$, and the relationship between vitamin D levels and AD severity. However, the results have been conflicting [1, 7-11].

Aim

In this study, we investigated the correlation between serum levels of 25 -hydroxyvitamin $\mathrm{D}\left(25(\mathrm{OH}) \mathrm{D}_{3}\right)$ and $A D$ severity.

\section{Material and methods}

Sixty patients (age range: $2-16$ years) who were diagnosed with $A D$ at an outpatient clinic, and followed regu-

Address for correspondence: Anil Gülsel Bahalı MD, Department of Dermatology, Medical Faculty, Bezmialem Vakif University, 34093 Fatih, Istanbul, Turkey, phone: +90 21245317 00-5912, +90 505 3893280, fax: +90 212 621 75 80, e-mail: anilirli@yahoo.com Received: 21.12.2015, accepted: 29.04.2016. 
larly from December 2013 to March 2015, were enrolled in the study. Clinical evaluation, the SCORAD index, and laboratory analysis results, including the level of total IgE, and $25(\mathrm{OH}) \mathrm{D}_{3}$ serum concentration, were collected retrospectively from the patients' records. Patients with missing records were excluded from the study. The control group consisted of 37 healthy subjects with no history or symptoms of allergic disease. The diagnosis of AD was based on the criteria of Hanifin and Rajka. The severity of AD was assessed by the same dermatologist using the scoring atopic dermatitis (SCORAD) index: mild AD (15), moderate AD (15-40), and severe AD (> 40). Although there is currently no consensus for optimal levels, vitamin D levels were categorized as sufficient (25-80 ng/ $\mathrm{ml}$ ), insufficient (10-24 $\mathrm{ng} / \mathrm{ml}$ ), or deficient (0-10 ng/ml). The patients and controls were also categorized according to season: winter/spring (December 1 to May 31) and summer/fall (June 1 to November 30).

The $25(\mathrm{OH}) \mathrm{D}_{3}$ serum concentrations in the $A D$ patients were compared with a control group. The level of vitamin D was evaluated in relationship to the severity of $A D$ and the total IgE. The age, sex, and seasonal characteristics of the groups in relationship to vitamin $D$ were investigated.

\section{Statistical analysis}

Specific information was presented as means, percentages, and standard deviations. Categorical variables were compared using the $\chi^{2}$ test, and the averages of the two subgroups were assessed with a t-test. When the effect of age was evaluated as a covariate, the same comparison was measured by a covariance test. Statistical analyses were conducted using IBM SPSS version 20.0. A $p$-value of 0.05 was considered significant.

\section{Results}

Sixty patients (30 males and 30 females) with $A D$ were enrolled in the study. The patients' age ranged between 2 and 16 years (mean: 8.37 years). The control group was composed of 37 patients (16 males and $21 \mathrm{fe}$ males), aged 2-14 years (mean: 6.54). The differences for age between the groups were eliminated by analysis of covariance. No significant relationship was found between the vitamin $\mathrm{D}$ level and age. The mean serum levels of vitamin $D$ in the patients with $A D$ and in the control group were $16.13 \pm 6.72$ and $19.76 \pm 10.4 \mathrm{ng} / \mathrm{ml}$, respectively, with no statistically significant difference between the groups ( $p=0.065)$.

With regard to the assessment according to season, 43 of the AD patients were enrolled in the winter/spring, and 17 were enrolled in the summer/fall. In the control group, 21 patients were enrolled in the winter/spring, and 16 in the summer/fall. There were no significant differences between the AD and control groups with respect to sex or seasonality. In the assessment of serum vita- min D levels according to season, there was a significant difference within the AD group, with mean levels in the winter/spring period much lower than for the summer/ fall period ( $p=0.008)$, but there were no differences in the control group $(p=0.855)$.

On the SCORAD examination, 31 patients had mild $A D, 20$ had moderate $A D$, and 6 had severe AD. A significant inverse relationship between SCORAD scores and vitamin $\mathrm{D}$ levels was found (Figure 1). Also, the relationship between vitamin D levels and SCORAD scores was assessed among patients with mild, moderate, and severe $A D$. The vitamin $D$ level was significantly lower in moderate and severe $A D$ compared with mild $A D$, and this difference was statistically significant ( $p=0.001$ and $p=0.004)$. However, there was no difference between the moderate and severe groups ( $p=0.992)$. The mean serum IgE level of the AD patients was 520.27, with a minimum of 1.00 and a maximum of 4,181 . Vitamin D showed a negative correlation with serum total lgE levels (Figure 2) ( $p=0.007)$. There was no significant correlation between total IgE levels and SCORAD scores ( $p=$ 0.089) (Figure 3).

\section{Discussion}

There have been several reports on the association between allergic disease and vitamin D. Recent data have shown that vitamin D affects immune mechanisms, keratinocytes, and skin barrier function. Vitamin $D$ is considered to play an important role in the development of allergic skin diseases, including $A D$, due to these effects. Although the majority of studies have demonstrated an inverse relationship between vitamin $\mathrm{D}$ levels and the prevalence or severity of $A D$, there is no consensus about the results of previous studies. Some studies have suggested that a high intake of vitamin D or an elevated serum vitamin $D$ level in infancy or during gestation can be associated with the development of eczema and asthma later in childhood. Thus, some studies have shown that vitamin $D$ is a protective factor for allergic disease, while others have shown that it is a risk factor for $A D$ [1, 12-14].

In the literature, Cheon et al., Wang et al., and Han et al. have reported that the level of $25(\mathrm{OH}) \mathrm{D}$ was significantly lower in patients with AD than in control groups. Samochocki et al. observed no statistically significant differences in the level of serum vitamin $D$ in adult $A D$ patients when compared with controls. Thuesen et al. investigated 2308 patients with an allergic disease such as asthma, atopy or atopic dermatitis. They found no significant associations between vitamin D and these allergic diseases. In our study, we found that vitamin D levels were lower in AD patients, but not statistically significantly [3, 9-11, 15].

A few studies have shown that vitamin D deficiency is associated with greater severity of $A D$, whereas others revealed no correlation. Peroni et al. reported an inverse 


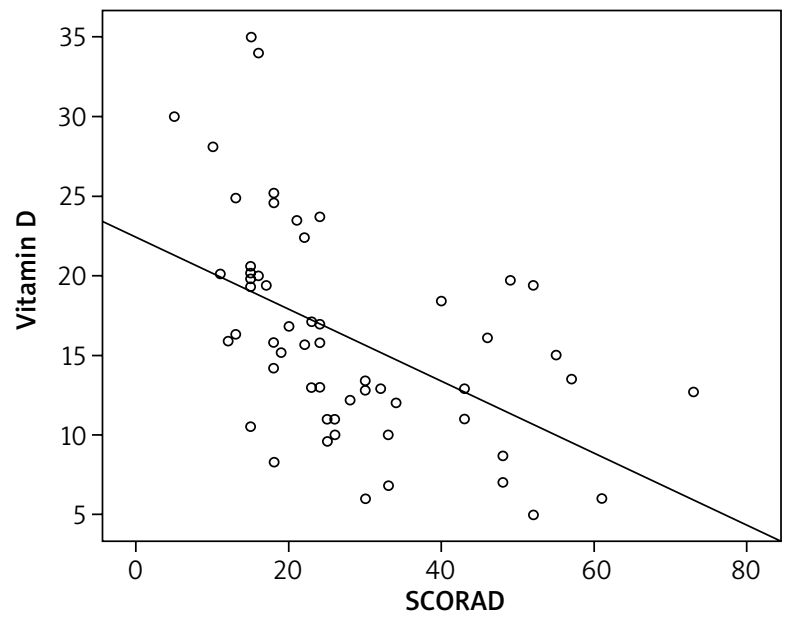

Figure 1. Inverse correlation between severity of atopic dermatitis and vitamin D

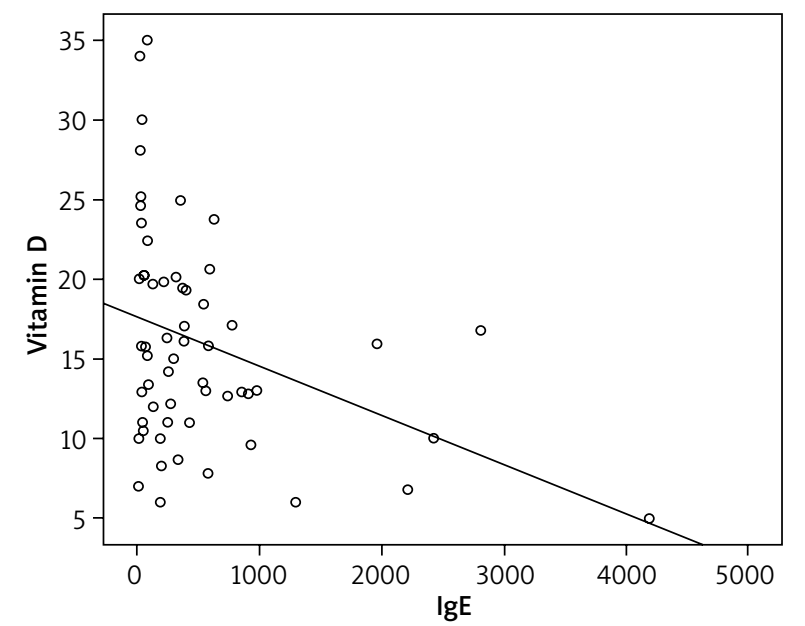

Figure 2. Inverse correlation between total lgE and vitamin D

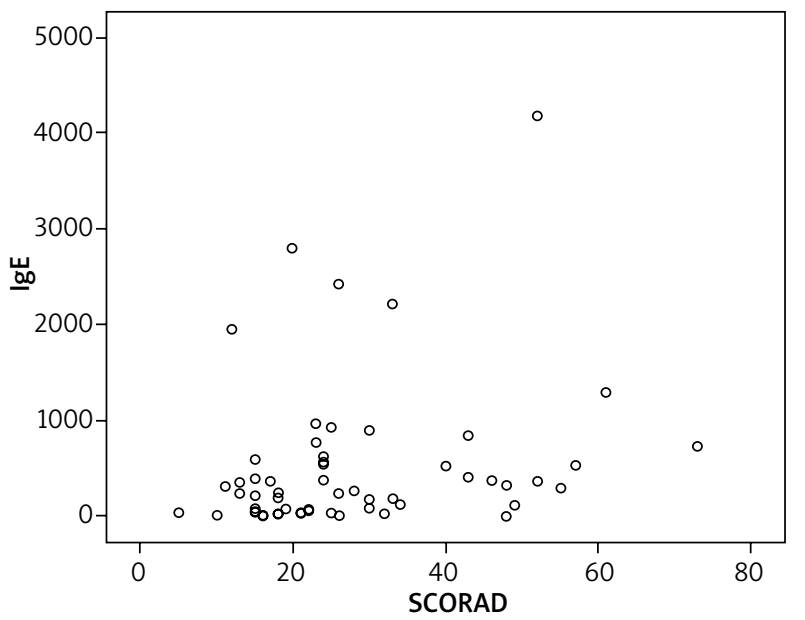

Figure 3. No correlation between SCORAD and IgE correlation between the serum concentration of $25(\mathrm{OH}) \mathrm{D}_{3}$ and severity of $A D$ in 37 children [16]. According to the results of Wang's study, the severity of $A D$ was inversely correlated with vitamin D deficiency in Chinese children living in Hong Kong. In contrast to our findings, Chiu et al. evaluated 94 children with $A D$, but they found no statistically significant correlation between vitamin D level and severity of disease [17]. The same results of no significant correlation were reported by Han et al. and by Samochocki et al. [3, 9-11, 16, 17].

In this study, we demonstrated that vitamin D levels were significantly lower in moderate and severe $A D$ compared with mild AD. This finding is the same as the results of El Taieb et al., who reported a negative correlation between vitamin $D$ and severity of AD. In fact, these results support our other findings as reported above [18].

Vitamin D has a significant role in Th1-Th2 balance. In addition, it has been reported that vitamin $\mathrm{D}$ receptor agonists suppress IgE production in cultured human B cells and in an allergy mouse model. Brehm et al. reported that lower vitamin D levels are associated with increased allergy markers, such as total IgE, in children with asthma. In studies on AD patients, Wang et al. and Cheon et al. found an inverse correlation between vitamin D and total IgE, similar to our findings [9, 10, 12, 19, 20].

We did not find a significant correlation between SCORAD and IgE. In contrast, Cheon et al. found that the SCORAD index was positively correlated with total IgE levels [10]. Total IgE may not be high in all patients with AD [21].

Several studies have reported an inverse association between sunlight exposure and AD severity. Thus, the severity of $A D$ might increase during the winter period. Vitamin D levels are affected by seasonal differences, and are much lower in winter than in summer because sunlight UVB exposure increases vitamin D serum levels. This idea is supported by clinical observations. In 2014, Camargo et al. investigated the role of oral vitamin $D$ supplementation in children with winter-related AD. They reported that this treatment led to clinical improvement in $\operatorname{AD}[2,7,22]$. In our study, patients who were evaluated during the winter/spring had higher SCORAD indices and lower vitamin $D$ levels. This result was not surprising due to much less exposure to solar radiation during this season.

There are studies that assessed the association between vitamin $D$ treatment and $A D$. The results are conflicting. Di Filippo et al. investigated 39 children with AD. The patients were treated with 1,000 IU/day ( $25 \mathrm{mg} /$ day) oral vitamin D for 3 months. They evaluated the vitamin D serum levels and SCORAD index before and after treatment. They reported that vitamin $D$ treatment may be an effective way for reducing $A D$ severity. Also, Amestejani et al. reported a randomized, double-blind, placebocontrolled study in which 60 patients with AD were enrolled. The patients were divided into two groups. One 
of the groups was treated with vitamin D (1,600 IU/day), the other was treated with placebo. After 60 days, they found that supplementation with oral vitamin D significantly improved disease severity in patients according to SCORAD and TIS (Three Item Severity score) [8, 23].

In the literature, different data were reported in the results of many studies. These conflicting results may be associated with geographic location, age, season, ethnicity, and environmental factors. Most vitamin D functions occur through vitamin D receptors (VDR). It is known that VDR may have effects on the regulation of the epidermal barrier function and cutaneous immune responses. Heine et al. showed that VDR gene polymorphisms in patients with severe AD. Hallau et al. reported that altered vitamin $D$ metabolism due to genetic variances have impacts on the pathogenesis of AD. VDR gene variations may result in altered responsiveness to vitamin D [2, 24, 25]. In addition, there were several limitations among previous studies on the relationship between vitamin $D$ and $A D$, such as a lack of standardization for serum vitamin D measurements. In addition, the diagnosis and severity of $A D$ was evaluated by different physicians, which may contribute to the differing results.

\section{Conclusions}

Vitamin $D$ has not been included in the routine treatment of AD because the conflicting results of various studies are still controversial. Certain studies in the literature, as well as our findings, have shown that there is an inverse correlation between vitamin D and the severity of AD. This is true especially during winter, when patients have lower vitamin D levels and higher SCORAD indices. Thus, we think that vitamin D supplementation for the treatment of AD would be useful, especially during the winter period. However, there is a need for more detailed and prospective studies.

\section{Conflict of interest}

The authors declare no conflict of interest.

\section{References}

1. Mesquita Kde C, Igreja AC, Costa IM. Atopic dermatitis and vitamin D: facts and controversies. An Bras Dermatol 2013; 88: 945-53.

2. Vestita M, Filoni A, Congedo $M$, et al. Vitamin D and atopic dermatitis in childhood. J Immunol Res 2015; 2015: 257879. doi: 10.1155/2015/257879.

3. Han TY, Kong TS, Kim MH, et al. Vitamin D status and its association with the SCORAD score and serum LL-37 level in Korean adults and children with atopic dermatitis. Ann Dermatol 2015; 27: 10-4.

4. Bergler-Czop B, Brzezińska-Wcisło L. Serum vitamin D level - the effect on the clinical course of psoriasis. Adv Dermatol Allergol 2016; 33: 445-9.

5. Karagün E, Ergin C, Baysak S, et al. The role of serum vitamin D levels in vitiligo. Adv Dermatol Allergol 2016; 33: 300-2.
6. Kucharska A, Szmurło A, Sińska B. Significance of diet in treated and untreated acne vulgaris. Adv Dermatol Allergol 2016; 33: 81-6.

7. Camargo CA Jr, Ganmaa D, Sidbury R, et al. Randomized trial of vitamin $D$ supplementation for winter-related atopic dermatitis in children. J Allergy Clin Immunol 2014; 134: 831-5.

8. Amestejani M, Salehi BS, Vasigh M, et al. Vitamin D supplementation in the treatment of atopic dermatitis: a clinical trial study. J Drugs Dermatol 2012; 11: 327-30.

9. Wang SS, Hon KL, Kong AP, et al. Vitamin D deficiency is associated with diagnosis and severity of childhood atopic dermatitis. Pediatr Allergy Immunol 2014; 25: 30-5.

10. Cheon BR, Shin JE, Kim YJ, et al. Relationship between serum 25-hydroxyvitamin D and interleukin-31 levels, and the severity of atopic dermatitis in children. Korean J Pediatr 2015; 58: $96-101$

11. Samochocki Z, Bogaczewicz J, Jeziorkowska R, et al. Vitamin D effects in atopic dermatitis. Am Acad Dermatol 2013; 69: 238-44.

12. Dębińska A, Sikorska-Szaflik H, Urbanik M, Boznański A. The role of vitamin D in atopic dermatitis. Dermatitis 2015; 26: $155-61$.

13. Gale CR, Robinson SM, Harvey NC, et al.; Princess Anne Hospital Study Group. Maternal vitamin D status during pregnancy and child outcomes. Eur J Clin Nutr 2008; 62: 68-77.

14. Bäck O, Blomquist HK, Hernell O, Stenberg B. Does vitamin $D$ intake during infancy promote the development of atopic allergy? Acta Dermatol Venereol 2009; 89: 28-32.

15. Thuesen BH, Heede NG, Tang L, et al. No association between vitamin $D$ and atopy, asthma, lung function or atopic dermatitis: a prospective study in adults. Allergy 2015; 70: 1501-4.

16. Peroni DG, Piacentini GL, Cametti E, et al. Correlation between serum 25-hydroxyvitamin D levels and severity of atopic dermatitis in children. Br J Dermatol 2011; 164: 1078-82.

17. Chiu YE, Havens PL, Siegel DH, et al. Serum 25-hydroxyvitamin D concentration does not correlate with atopic dermatitis severity. J Am Acad Dermatol 2013; 69: 40-6.

18. El Taieb MA, Fayed HM, Aly SS, Ibrahim AK Assessment of serum 25-hydroxyvitamin D levels in children with atopic dermatitis: correlation with SCORAD index. Dermatitis 2013; 24: 296-301.

19. Hartmann B, Heine G, Babina M, et al. Targeting the vitamin $D$ receptor inhibits the $B$ cell-dependent allergic immune response. Allergy 2011; 66: 540-8.

20. Brehm JM, Celedón JC, Soto-Quiros ME, et al. Serum vitamin D levels and markers of severity of childhood asthma in Costa Rica. Am J Respir Crit Care Med 2009; 179: 765-71.

21. Ahmed I, Nasreen S. Frequency of raised serum IgE level in childhood atopic dermatitis. J Pak Med Assoc 2007; 57: 431-4.

22. Vähävihu K, Ala-Houhala M, Peric M, et al. Narrowband ultraviolet $B$ treatment improves vitamin $D$ balance and alters antimicrobial peptide expression in skin lesions of psoriasis and atopic dermatitis. Br J Dermatol 2010; 163: 321-8.

23. Di Filippo P, Scaparrotta A, Rapino D, et al. Vitamin D supplementation modulates the immune system and improves atopic dermatitis in children. Int Arch Allergy Immunol 2015; 166: 91-6.

24. Heine G, Hoefer N, Franke A, et al. Association of vitamin D receptor gene polymorphisms with severe atopic dermatitis in adults. Br J Dermatol 2013; 168: 855-8.

25. Hallau J, Hamann L, Schumann RR, et al. A promoter polymorphism of the vitamin D metabolism gene Cyp24a1 is associated with severe atopic dermatitis in adults. Acta Derm Venereol 2016; 96: 169-72. 